\section{RICYDE. Revista Internacional de Ciencias del Deporte doi: $10.5232 /$ ricyde \\ Rev. Int. cienc. deporte}

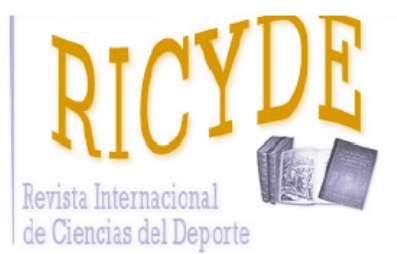

RICYDE. Revista Internacional de Ciencias del Deporte Volumen XIV - Año XIV

Páginas: 358-373 - ISSN: 1885-3137

Número 54 - octubre 2018

\title{
Asimetrías neuromusculares entre miembros inferiores en jugadores de hockey sobre patines Lower limb neuromuscular asymmetry in roller hockey players
}

\author{
Jordi Arboix-Alión ${ }^{1,2}$, Joan Aguilera-Castells ${ }^{2}$, Fernando Rey-Abella ${ }^{1}$, Bernat Buscà ${ }^{2}$, \\ y Azahara Fort-Vanmeerhaeghe ${ }^{1,2}$
}

1. Facultat de Ciències de la Salut Blanquerna (FCS), Universitat Ramon Llull, Barcelona, España
2. Facultat de Psicologia, Ciències de I'Educació i de I'Esport Blanquerna (FPCEE), Universitat Ramon Llull, Barcelona, España

\section{Resumen}

El objetivo de este trabajo fue comparar y cuantificar las asimetrías neuromusculares entre las extremidades inferiores que presentan los jugadores de hockey sobre patines. Un objetivo secundario fue correlacionar el índice de asimetrías (ASI) entre las diferentes pruebas neuromusculares testadas. Un total de 27 jugadores de sexo masculino de hockey sobre patines (edad $=20,59 \pm 3,81$ años) realizaron diferentes pruebas neuromusculares de forma unilateral: 1) Test de salto vertical con contra-movimiento (SLCJ), 2) Batería de saltos horizontales (Hop test: single, triple y crossover hop test) y 3) Prueba de velocidad con cambio de dirección (COD test). Se observaron diferencias significativas $(p<0,05)$ al comparar el rendimiento de las extremidades inferiores para todas las capacidades neuromusculares testadas. La media del ASI entre miembros inferiores de los jugadores osciló entre el 4,93\% y el 9,60\% en función del test, siendo máxima en el SLC] $(9,60 \%)$. En un 33,3\% de los jugadores de la muestra se observó un ASI $>10 \%$ en el test de SLC], situación considerada como posible factor de riesgo de lesión. Solamente se obtuvo una correlación significativa entre el SLC] y el COD test. En consecuencia, y con el objetivo de identificar las asimetrías entre miembros inferiores, es importante considerar la valoración de las diferencias capacidades neuromusculares.

Palabras clave: desequilibrios neuromusculares; prevención lesiones; deporte de equipo; evaluación funcional.

\section{Abstract}

The aim of the current study was to compare and quantify the neuromuscular lower limb asymmetries present in roller hockey players and its link to high risk of injury. A secondary purpose was to correlate the index of asymmetries (ASI) between the different neuromuscular tests performed. 27 male roller hockey players (age $=20.59 \pm 3.81$ years) performed the single leg countermovement jump (SLCJ), the Hop Test battery (single hop test, triple hop test and crossover hop test) and the change of direction test (COD test). Significant differences were observed $(p<0.05)$ between the lower limb established according to the objective criterion of dominant and non-dominant leg for all tasks. The average difference of the ASI between players' legs ranges from $4.93 \%$ to $9.60 \%$ depending on the test, being maximum in the single leg countermovement jump (9.60\%). A $33.3 \%$ of the players in the sample obtained an ASI> $10 \%$ in the $\mathrm{SLCJ}$, a situation considered as an injury risk factor. Only a significant correlation was obtained between the SLCJ and the COD test. Consequently, it is suggested that the complete neuromuscular profile between lower limbs be evaluated with the different tests, for both in the conditioning programs and in the injury prevention and return-to- play.

Key words: neuromuscular imbalances; injury prevention; team sport; functional evaluation.

Correspondencia/correspondence: Jordi Arboix-Alió

Facultat de Ciències de la Salut Blanquerna (FCS), Universitat Ramon Llull, Barcelona, España

Email: jordiaa1@blanquerna.url.edu 
Arboix-Alió, J.; Aguilera-Castells, J.; Rey-Abella, F.; Buscà, B., y Fort-Vanmeerhaeghe, A. (2018). Asimetrías neuromusculares entre miembros inferiores en jugadores de hockey sobre patines. RICYDE. Revista internacional de ciencias del deporte. 54(14), 358-373. https://doi.org/10.5232/ricyde2018.05406

\section{Introducción}

A ctualmente, existe una gran incidencia lesiva tanto en el deporte profesional como en el deporte amateur, lo que supone un perjuicio económico importante tanto a nivel de clubes como de costes sanitarios de un país (García, Albaladejo, Villanueva, y Navarro, 2015).

Las lesiones deportivas tienen una causa multifactorial que está enumerada en varias clasificaciones. Murphy, Connolly, y Beynnon (2003) diferencian los factores de riesgo en extrínsecos e intrínsecos al deportista. Los primeros hacen referencia a los elementos externos como son el equipamiento utilizado o las condiciones ambientales, mientras que los segundos hacen referencia a aquellos elementos propios del deportista como la edad, el sexo, la composición corporal o sus características anatómicas y capacidades físicas. Otro tipo de clasificación sería la que divide la lesión en 4 categorías: ambiental, anatómica, hormonal y neuromuscular; destacando esta última categoría como la más influenciable por el entrenamiento (Griffin y col., 2006). Dentro de los factores de riesgo neuromusculares destacarían los desequilibrios neuromusculares entre pierna dominante (PD) y pierna no dominante (PND) (Fort-Vanmeerhaeghe, Romero-Rodriguez, Montalvo, y col., 2016). Asimismo, es importante destacar que los desequilibrios neuromusculares entre miembros inferiores suelen ser mayores en las deportistas femeninas (Fort-Vanmeerhaeghe, Gual, Romero-Rodriguez, y Unnitha, 2016). Estas diferencias entre extremidades pueden ser aún más determinantes en deportistas que han sufrido una lesión, facilitando la recidiva; por ejemplo en lesiones del ligamento cruzado anterior (Paterno y col., 2010) o del ligamento lateral externo del tobillo (Ross y Guskiewicz, 2004).

Habitualmente la denominación de la PD y la PND se ha establecido de forma subjetiva según diversos criterios. Algunos autores la definen como la pierna con la que el sujeto manifiesta una mayor maestría para realizar habilidades básicas como disparos o controles de balón (Meylan y col., 2009; Miyaguchi y Demura, 2010; Theoharopoulos, Tsitskaris, Nikopoulou, y Tsaklis, 2000) o como la pierna con la que se inicia el movimiento de subir un escalón (Ceroni, Martin, Delhumeau, y Farpour-Lambert, 2012). Sin embargo, varios autores han apuntado que muchos sujetos no saltan más alto ni más lejos con la PD (FortVanmeerhaeghe, Montalvo, Sitjà-Rabert, Kiefer, y Myer, 2015; Schiltz y col., 2009; Stephens y col., 2007; Troule y Casamichana, 2016) y que este criterio no es suficientemente fiable para detectar diferencias en las capacidades neuromusculares (Rahnama y col., 2005; Schiltz y col., 2009; Stephens, Lawson, DeVoe, y Reiser, 2007). Por este motivo otro criterio de clasificación más preciso ha sido el que clasifica objetivamente la PD y la PND según la que obtiene un mayor rendimiento en las habilidades de saltos o cambios de dirección. Para evaluar los desequilibrios neuromusculares entre PD y PND, se utiliza el índice de asimetría (ASI) (Ceroni y col., 2012; Overmoyer y Reiser, 2013). Según las evidencias actuales, una diferencia entre miembros inferiores del $10-15 \%$ en el rendimiento en pruebas de fuerza isocinética y de salto vertical puede incrementar el riesgo de lesión (Hewit, Cronin, y Hume, 2012; McElveen, Riemann, y Davies, 2010; Munro y Herrington, 2011).

Tradicionalmente, para medir las asimetrías entre la extremidad inferior dominante y no dominante, se han empleado pruebas isocinéticas (Maulder y Cronin, 2005; Rahnama, Lees, y Bambaecichi, 2005; Ross, Guskiewicz, Prentice, Schneider, y Yu, 2004). Sin embargo, dada la poca funcionalidad de estas pruebas con el gesto deportivo y el hecho de que requieran de un equipamiento con un alto coste económico, se han dejado de utilizar en el ámbito del entrenamiento y rendimiento deportivo en detrimento de otros test más funcionales y económicos. Algunos de estos test que reproducen mejor el gesto deportivo y por lo tanto son 
Arboix-Alió, J.; Aguilera-Castells, J.; Rey-Abella, F.; Buscà, B., y Fort-Vanmeerhaeghe, A. (2018). Asimetrías neuromusculares entre miembros inferiores en jugadores de hockey sobre patines. RICYDE. Revista internacional de ciencias del deporte. 54(14), 358-373. https://doi.org/10.5232/ricyde2018.05406

más específicos, son los test que evalúan la capacidad de salto unilateral, método empleado en varios estudios para valorar las asimetrías entre extremidades (Ceroni y col., 2012; FortVanmeerhaeghe, Gual y col., 2016; Fort-Vanmeerhaeghe y col., 2015; Meylan y col., 2009; Troule y Casamichana, 2016). Parece ser que el motivo principal para utilizar los test de salto unilateral se fundamenta en la predominancia de las acciones unilaterales presentes en la mayoría de deportes donde se requiere el ciclo de estiramiento-acortamiento para generar potencia (Ceroni y col., 2012). Los test de salto unilateral más conocidos son el test "Single Leg Countermovement Jump" (SLCJ) que mide el componente de salto vertical y la batería "Hop Test", que mide el componente de salto horizontal para valorar la fuerza muscular y el control neuromuscular (Noyes, Barber, y Mangine, 1991). Estos test son muy utilizados en el ámbito deportivo para determinar el estado físico del deportista, especialmente durante el proceso de rehabilitación posterior a una lesión (Brumitt, Heiderscheit, Manske, y Niemuth, 2013; Troule y Casamichana, 2016), pero también en deportistas sanos a fin de detectar asimetrías de la extremidad inferior (Fort-Vanmeerhaeghe, Gual y col., 2016).

Actualmente la literatura científica nos muestra distintas investigaciones que examinan las asimetrías neuromusculares en deportes individuales como el tenis (Sannicandro, Cofano, Rosa, y Piccinno, 2014), la gimnasia rítmica (Batista, Bobo, Lebre, y Ávila-Carvalho, 2015; Simas Frutuoso, Diefenthaeler, Vaz, y de la Rocha Freitas, 2016) o las carreras de ultra fondo (Dellagrana, Diefenthaeler, Carpes, Hernandez, y de Campos, 2015). Sin embargo, la gran mayoría de estudios se centran en los deportes colectivos; especialmente el fútbol (Daneshjoo, Rahnama, Mokhtar, y Yusof, 2013; Dauty y col., 2016; Hart y col., 2016; Menzel y col., 2013; Troule y Casamichana, 2016). Asimismo las asimetrías neuromusculares también se han estudiado en el baloncesto (Fort-Vanmeerhaeghe y col., 2015; Sugiyama y col., 2014), el balonmano (Pontaga y Zidens, 2014), el rugby (Marshall y col., 2015), el hockey hierba (Krzykała y Leszczyński, 2015) o el voleibol (Castanharo y col., 2011; FortVanmeerhaeghe, Gual y col., 2016; Markou y Vagenas, 2006). A pesar de los numerosos estudios que examinan las asimetrías neuromusculares en la extremidad inferior en deportes colectivos, por lo que sabemos, no se han encontrado estudios que muestren evidencias sobre asimetrías neuromusculares en hockey sobre patines. Este deporte presenta muchas acciones técnicas que se desarrollan de manera unilateral. Cómo en otros deportes colectivos, el hecho de realizar acciones donde se combina el componente explosivo y de unilateralidad como cambios de ritmo o de dirección hace que estos deportistas puedan desarrollar adaptaciones neuromusculares asimétricas (Fort-Vanmeerhaeghe, Gual y col., 2016; Yanci y Camara, 2016), aspecto que podría comportar para el deportista tener un mayor riesgo de incidencia lesiva (Daneshjoo y col., 2013; Fort-Vanmeerhaeghe y col., 2009; Fort-Vanmeerhaeghe y Romero, 2013). En relación a las lesiones en el hockey sobre patines se han encontrado pocos estudios que analizan las lesiones específicas de este deporte (Pons y Ferrer, 1991; Varlotta, Lager, Nicholas, Browne, y Schlifstein, 2000).

En la actualidad no se dispone de datos ni de estudios que traten las asimetrías neuromusculares en jugadores de hockey sobre patines. Por lo tanto, una de las dudas existentes es si los jugadores de hockey sobre patines presentan asimetrías neuromusculares entre miembros inferiores. Debido a la controversia y falta de información científica al respecto, se plantearon los siguientes objetivos: 1) Comparar y cuantificar las asimetrías neuromusculares entre las extremidades inferiores (capacidad de salto unilateral y cambios de dirección); 2) Correlacionar el índice de asimetrías entre las diferentes pruebas neuromusculares testadas. La principal hipótesis es que, en el hockey sobre patines, al ser un deporte asimétrico donde predominan las acciones unilaterales, existirán asimetrías significativas entre piernas, similares a otros deportes de equipo. Y de forma secundaria, que 
Arboix-Alió, J.; Aguilera-Castells, J.; Rey-Abella, F.; Buscà, B., y Fort-Vanmeerhaeghe, A. (2018). Asimetrías neuromusculares entre miembros inferiores en jugadores de hockey sobre patines. RICYDE. Revista internacional de ciencias del deporte. 54(14), 358-373. https://doi.org/10.5232/ricyde2018.05406

no habrá una correlación significativa entre los resultados de los índices de asimetrías entre piernas (\%ASI) obtenidos en las diferentes pruebas.

\section{Método}

\section{Participantes}

La muestra estuvo configurada por un total de 27 jugadores de hockey sobre patines de sexo masculino (edad $=20.59 \pm 3.81$ años). Todos los jugadores debían entrenar un mínimo de tres veces por semana (aproximadamente 6 a 10 horas/semana), 8-9 meses al año y jugar como mínimo un partido cada fin de semana durante toda la temporada. Los criterios de exclusión fueron cualquier lesión (aguda o crónica) o enfermedad en el momento de las pruebas que impidieran llevar a cabo los test al máximo rendimiento. Antes del inicio del estudio, todos los sujetos firmaron el consentimiento informado voluntariamente, habiéndose leído previamente el documento de información de los participantes. El diseño de la investigación se ajustó a lo establecido por la Declaración de Helsinki y fue aprobado por el Comité de Ética de la Universidad Ramon Llull de Barcelona. Además, el departamento técnico del club proporcionó su consentimiento para la realización del estudio.

\section{Técnicas instrumentales}

La investigación se realizó durante el periodo de competición, dentro del horario habitual de entrenamiento (21.00 horas), durante una sesión de entrenamiento después de un día de descanso y posterior a 72 horas del partido anterior. Se realizó un calentamiento estandarizado de 15 minutos (carrera continua de moderada intensidad, ejercicios de movilidad articular y cambios de velocidad progresivos a máxima intensidad) previo al inicio del test. Todos los test se llevaron a cabo en una pista polideportiva cubierta ( 44 x $22 \mathrm{~m}$ ) de pavimento de parquet. Los sujetos no ingirieron bebidas alcohólicas, ni cafeína, ni realizaron actividad física intensa las $24 \mathrm{~h}$ previas a los test. Tampoco se permitió la ingesta de alimentos dos horas antes de la realización de los mismos.

Se realizaron tres repeticiones con cada extremidad inferior en cada test, primero con la descrita subjetivamente como dominante y luego con la no dominante. No se repitió ningún intento con la misma pierna sin respetar un descanso de 30 segundos. La PD fue descrita de forma subjetiva como la pierna con la que chutarían una pelota. Para evitar la fatiga, el tiempo de recuperación entre test fue de 2 minutos. Cuando el deportista realizaba alguna repetición considerada nula, se repetía de nuevo la prueba hasta que se realizara correctamente, respetando la recuperación pertinente. Para el análisis de datos se escogió el promedio de los tres intentos realizados con cada test. El orden de los test y de los participantes fueron aleatorizadas con el programa "True random number generator" (Haahr, 2017).

\section{$\underline{\text { Salto unilateral con contra-movimiento (SLCJ) }}$}

La capacidad de salto vertical unilateral de los participantes se avaluó mediante el test de salto unilateral con contra-movimiento, denominado en lengua anglosajona "Single Leg Countermovement Jump"(SLCJ), y siguiendo a los autores Meylan y col., (2009). Se calculó el tiempo de vuelo según el protocolo de Bosco (de Blas, Padullés, López del Amo, y GuerraBalic, 2012) con una plataforma de contactos (Chronojump-Boscosystem ${ }^{\circledR}$, Barcelona, ESP).

En este test se indicó a los sujetos que realizaran un salto vertical con una pierna tras un rápido contra-movimiento hacia abajo (Fig. 1). Durante la acción de flexión de rodillas y cadera, el tronco debía permanecer lo más recto posible para evitar cualquier posible influencia del mismo en el rendimiento de los miembros inferiores. También se les indicó 
Arboix-Alió, J.; Aguilera-Castells, J.; Rey-Abella, F.; Buscà, B., y Fort-Vanmeerhaeghe, A. (2018). Asimetrías neuromusculares entre miembros inferiores en jugadores de hockey sobre patines. RICYDE. Revista internacional de ciencias del deporte. 54(14), 358-373. https://doi.org/10.5232/ricyde2018.05406

que aterrizaran simultáneamente con ambos pies. El test se consideró correcto si las manos permanecían en las caderas durante todo el movimiento y el equilibrio se mantuvo durante al menos tres segundos después del aterrizaje. Estas tareas han demostrado tener una fiabilidad excelente (Hewit y col., 2012; Maulder y Cronin, 2005; Meylan y col., 2009)
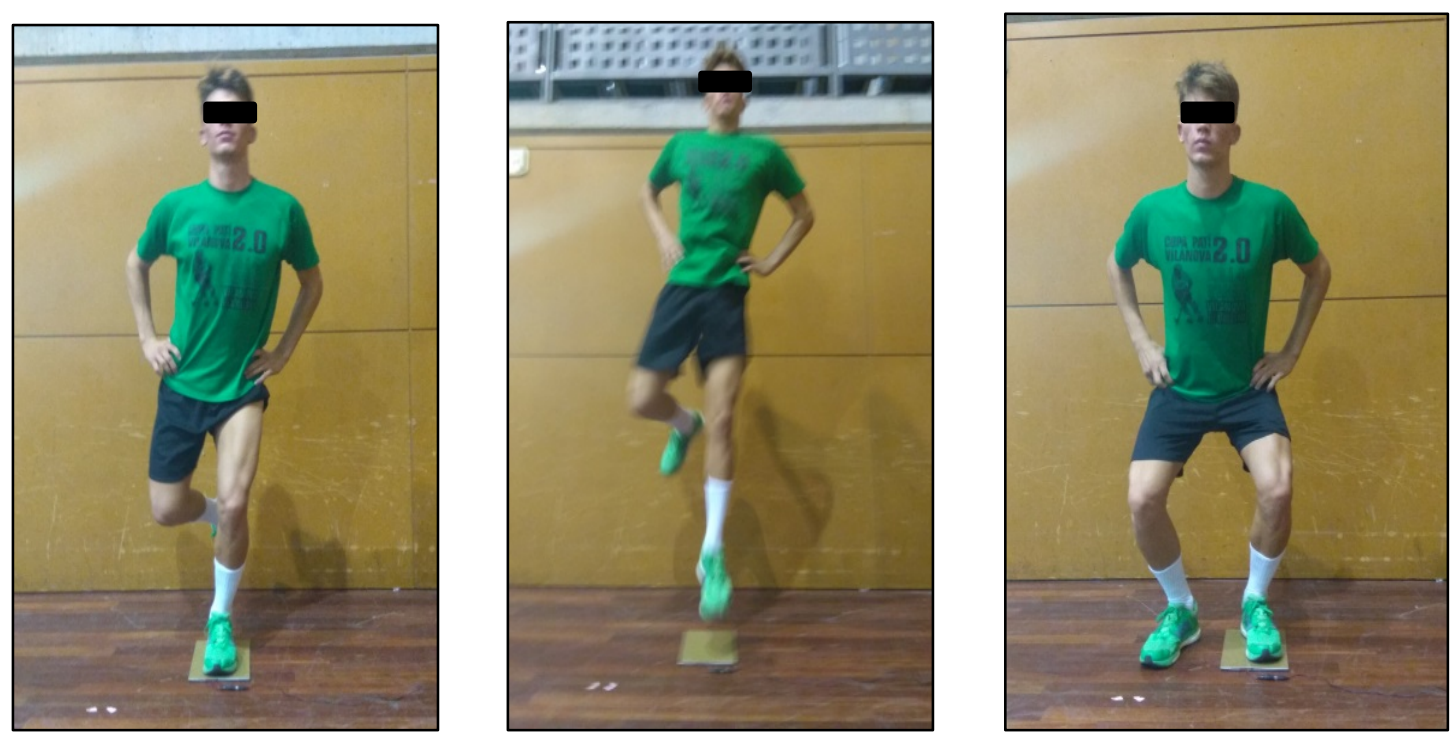

Figura 1. Test del Single leg Counter Movement Jump.

Batería de saltos unilaterales horizontales

Consiste en una batería de test de saltos horizontales unilaterales, también denominados en lengua anglosajona "Hop test", para calcular la asimetría entre extremidades inferiores (Noyes y col., 1991). En la presente investigación se han realizado los siguientes tres test (Fig. 2) que han demostrado ser los más fiables y válidos dentro del conjunto de pruebas de los Hop Test (Logerstedt, Snyder-Mackler, Ritter, Axe, y Godges, 2010; Reid, Birmingham, Stratford, Alcock, y Giffin, 2007).

Single Hop test: consiste en hacer un salto partiendo de un apoyo monopodal y caer con la misma pierna que se realiza el impulso. Se cuantifica la distancia alcanzada.

Triple Hop test: consiste en enlazar tres saltos sobre el mismo apoyo, partiendo desde un contacto monopodal y finalizando los tres saltos sobre la misma pierna. Se cuantifica la distancia alcanzada.

Crossover Hop test: igual que en el triple hop test, deben enlazarse tres saltos con idéntica dinámica de inicio y finalización, pero cada uno de los apoyos se realiza a un lado de una línea. Se cuantifica la distancia alcanzada.

Para los tres test, se indicó a los sujetos que debían colocar las manos sobre las caderas y que la posición de aterrizaje debía mantenerse durante 2-3 s, sin pérdida de equilibrio ni realizar movimientos adicionales que implicasen a la extremidad libre. La distancia fue medida en centímetros desde la línea de batida hasta el talón en la posición final. Los tres test han demostrado una buena fiabilidad en el test-retest en adultos jóvenes sanos (Munro y Herrington, 2011). 
Arboix-Alió, J.; Aguilera-Castells, J.; Rey-Abella, F.; Buscà, B., y Fort-Vanmeerhaeghe, A. (2018). Asimetrías neuromusculares entre miembros inferiores en jugadores de hockey sobre patines. RICYDE. Revista internacional de ciencias del deporte. 54(14), 358-373. https://doi.org/10.5232/ricyde2018.05406

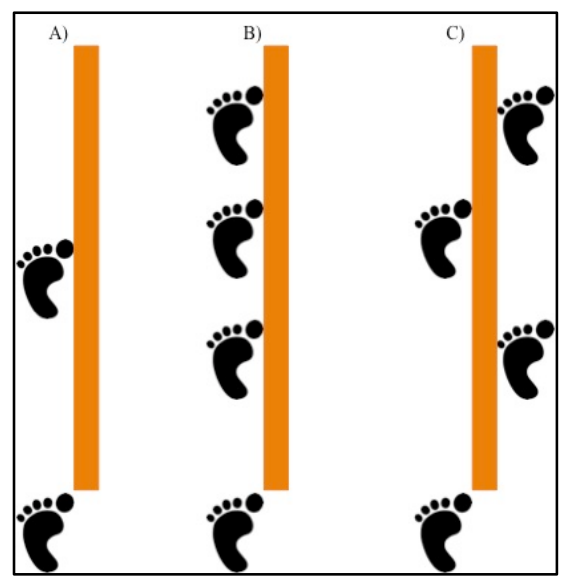

Figura 2. Batería Hop Test. A) Single Hop Test, B) Triple Hop Test, C) Crossover Hop Test.

\section{Cambio de dirección (COD).}

El tiempo empleado en ejecutar el COD test fue calculado con una fotocélula (Vellman PED 10D; Düsseldorf, DEU) conectada a un ordenador (Chronojump-Boscosystem ${ }^{\circledR}$, Barcelona, ESP) (de Blas y col., 2012). Se indicó a los sujetos que debían recorrer 5 metros lo más rápidamente posible, pivotar con una pierna y volver a recorrer 5 metros hasta la posición inicial (Fig. 3). Este test, se realizó primeramente pivotante con una pierna y luego pivotante con la otra. Para considerar la ejecución válida, el sujeto debe pisar la línea. Por otro lado, vista la poca correlación entre los desplazamientos efectuados en patines y zapatillas deportivas en jugadores de hockey y siguiendo las recomendaciones de Arboix-Alió, Aguilera-Castells, y Ferrándiz Bernal (2017) se realiza la prueba con patines. Esta prueba tiene una buena fiabilidad test-retest (Calleja-González y col., 2015; Castillo-Rodríguez, Fernández-García, Chinchilla-Minguet, y Carnero, 2012).
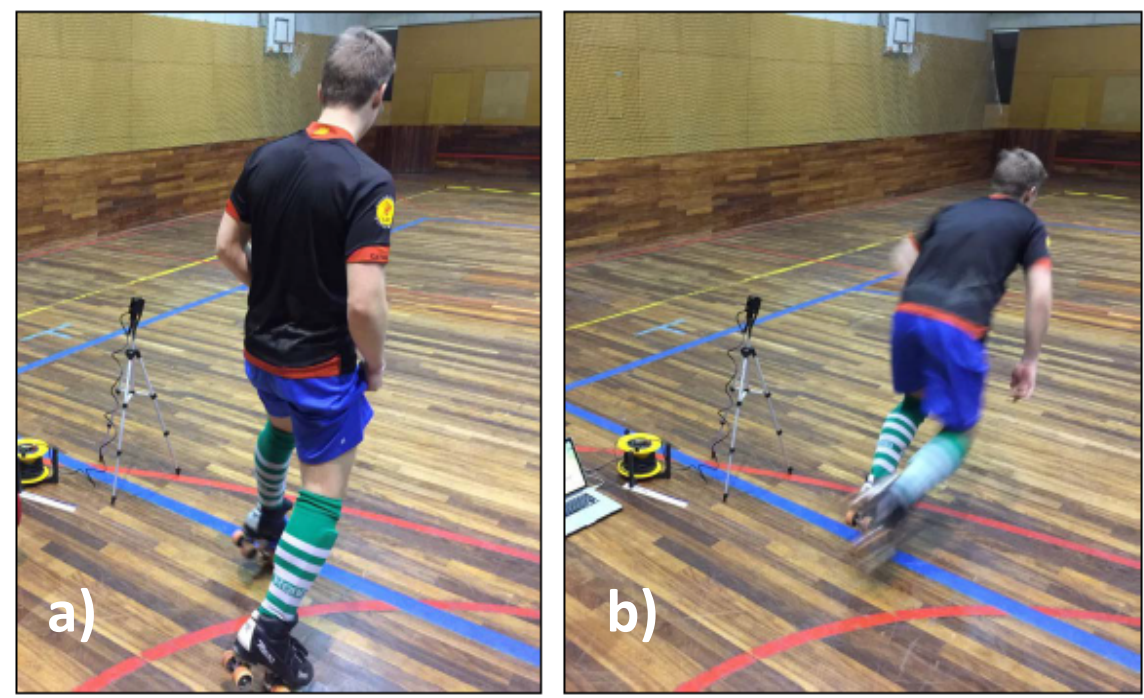

Figura 3. Test de Cambio de Dirección con patines. La imagen de la izquierda (a) muestra la posición inicial del test, y la imagen de la derecha (b) muestra el momento de inicio del test justo cuando el jugador corta el haz luminoso de la fotocélula. 
Arboix-Alió, J.; Aguilera-Castells, J.; Rey-Abella, F.; Buscà, B., y Fort-Vanmeerhaeghe, A. (2018). Asimetrías neuromusculares entre miembros inferiores en jugadores de hockey sobre patines. RICYDE. Revista internacional de ciencias del deporte. 54(14), 358-373. https://doi.org/10.5232/ricyde2018.05406

\section{Procedimiento}

Se realizó un estudio transversal para comparar el rendimiento neuromuscular unilateral en un grupo de jugadores de hockey patines. Los jugadores $(n=27)$ se dividieron en dos subgrupos (Grupo1, n=13) y (Grupo2, n=14). La distribución de estos en los dos grupos fue aleatorizada. Los participantes empezaron realizando los test de salto (Batería "Hop Test", y SLCJ) y seguidamente el COD test. Entre test se establecieron 2 minutos de recuperación para evitar la fatiga. Se comparó la altura de salto vertical unilateral de miembros inferiores, la distancia de salto horizontal unilateral y el tiempo obtenido en el COD test. Se analizó el ASI y secundariamente se valoró si la PD asumía un mayor registro en comparación con la no dominante en las distintas variables dependientes estudiadas.

\section{Análisis estadístico}

Se utilizaron métodos estadísticos descriptivos para describir la muestra y para calcular los promedios y desviaciones estándar de las diferentes variables analizadas. Para comprobar la normalidad de la muestra se llevó a cabo el test de hipótesis de Shapiro-Wilk para muestras inferiores a 50 sujetos. Se utilizó la prueba t-Student para muestras relacionadas para establecer si existían diferencias significativas entre ambas piernas en los diferentes test.

Con el fin de identificar la asimetría neuromuscular entre los miembros se comparó la pierna con mayor rendimiento (PD) con la que obtuvo un menor rendimiento (PND) y se calculó el ASI utilizando la siguiente fórmula (Carpes, Mota, y Faria, 2010; Impellizzeri, Rampinini, Maffiuletti, y Marcora, 2007):

\section{ASI = (pierna dominante - pierna PND / pierna dominante) $\times 100$}

La prueba de Pearson se utilizó para determinar el grado de correlación de los valores obtenidos en el índice ASI en los distintos test neuromusculares. El nivel de significación se determinó en $\mathrm{p}<0,05$. El análisis estadístico se realizó con el programa estadístico SPSS (Versión 20.0 para Mac; SPSS Inc., Chicago, IL, USA).

\section{Resultados}

Las características de los sujetos se presentan en la Tabla 1.

Se encontraron diferencias significativas entre PD y PND en todas las pruebas registradas (COD, "Hop Test" y SLCJ) (Tabla 2). Los valores medios de ASI variaron entre el 4,93\% y el $9,60 \%$ dependiendo del test. La Tabla 3 muestra el porcentaje de jugadores con un \% ASI superior al 10-15\%, considerado un posible factor de riesgo de lesiones deportivas (Hewit y col., 2012; McElveen y col., 2010).

En la Tabla 4 se muestra una matriz de correlación entre los diferentes test neuromusculares y los valores del ASI. Solo se obtuvo una correlación significativa entre el ASI del SLCJ y el COD test $(\mathrm{p}<0,05)$. 
Arboix-Alió, J.; Aguilera-Castells, J.; Rey-Abella, F.; Buscà, B., y Fort-Vanmeerhaeghe, A. (2018). Asimetrías neuromusculares entre miembros inferiores en jugadores de hockey sobre patines. RICYDE. Revista internacional de ciencias del deporte. 54(14), 358-373. https://doi.org/10.5232/ricyde2018.05406

Tabla 1. Características de los sujetos. Datos expresados en media $\pm \mathrm{SD}$.

\begin{tabular}{ll}
\hline Edad (años) & $20,59 \pm 3,81$ \\
Altura $(\mathrm{m})$ & $1,77 \pm 0,08$ \\
Peso $(\mathrm{kg})$ & $71,53 \pm 11,74$ \\
$\mathrm{IMC}$ & $22,87 \pm 3,06$ \\
\% grasa & $12,18 \pm 4,62$ \\
Derecha/Izquierda PD & $23 / 4$ \\
\hline
\end{tabular}

IMC_Índice de Massa Corporal; Derecha/ Izquierda PD_Pierna dominante descrita de forma subjetiva, es decir la pierna con la que chutarían una pelota

Tabla 2. Comparación de los resultados en los test de salto y cambio de dirección según la extremidad inferior. Los datos se muestran en media \pm SD.

\begin{tabular}{|c|c|c|c|c|c|}
\hline & $\begin{array}{c}\text { SINGLE } \\
\text { HOP TEST } \\
(\mathrm{m})\end{array}$ & $\begin{array}{l}\text { TRIPLE HOP } \\
\text { TEST (m) }\end{array}$ & $\begin{array}{c}\text { CROSSOVER } \\
\text { TEST (m) }\end{array}$ & $\begin{array}{l}\text { COD (se- } \\
\text { gundos) }\end{array}$ & $\begin{array}{l}\text { SLCJ } \\
(\mathrm{cm})\end{array}$ \\
\hline P. Dominante & $1,83 \pm 0,16$ & $5,99 \pm 0,51$ & $5,68 \pm 0,67$ & $2,83 \pm 0,20$ & $17,69 \pm 4,04$ \\
\hline P. No Dominante & $1,71 \pm 0,17$ & $5,69 \pm 0,43$ & $5,33 \pm 0,61$ & $3,01 \pm 0,18$ & $15,96 \pm 3,78$ \\
\hline $\mathrm{p}$ & $0,000 *$ & $0,000 *$ & $0,000 *$ & $0,000 *$ & $0,000 *$ \\
\hline$\eta_{\mathrm{p}}^{2}$ & 0,73 & 0,64 & 0,55 & $-0,95$ & 0,44 \\
\hline ASI (\%) & 6,35 & 4,93 & 5,82 & 6,45 & 9,60 \\
\hline
\end{tabular}

Tabla 3. Número y porcentaje de jugadores con un ASI $>10 \%$.

\begin{tabular}{lccccc}
\hline & $\begin{array}{c}\text { SINGLE } \\
\text { HOP TEST }\end{array}$ & $\begin{array}{c}\text { TRIPLE } \\
\text { HOP TEST }\end{array}$ & $\begin{array}{c}\text { CROSSOVER } \\
\text { TEST }\end{array}$ & COD & SLCJ \\
\hline $\begin{array}{l}\text { P. Dominante vs. } \\
\text { P. No dominante }\end{array}$ & $5(18,51 \%)$ & $3(11,11 \%)$ & $5(18,51 \%)$ & $5(18,51 \%)$ & $9(33,33 \%)$ \\
\hline $\begin{array}{l}\text { P_Pierna; COD_Cambio de dirección; SLCJ_Salto vertical unilateral con contra-movimiento; ASI_ Índice de } \\
\text { asimetría (\%) }\end{array}$ & & & \\
\hline
\end{tabular}


Arboix-Alió, J.; Aguilera-Castells, J.; Rey-Abella, F.; Buscà, B., y Fort-Vanmeerhaeghe, A. (2018). Asimetrías neuromusculares entre miembros inferiores en jugadores de hockey sobre patines. RICYDE. Revista internacional de ciencias del deporte. 54(14), 358-373. https://doi.org/10.5232/ricyde2018.05406

Tabla 4. Correlación del índice ASI (\%) entre los diferentes test neuromusculares.

\begin{tabular}{crrrrr}
\hline & $\begin{array}{r}\text { SINGLE } \\
\text { HOP TEST }\end{array}$ & $\begin{array}{r}\text { TRIPLE } \\
\text { HOP TEST }\end{array}$ & $\begin{array}{r}\text { CROSSOVER } \\
\text { TEST }\end{array}$ & COD & SLCJ \\
\hline SINGLE HOP TEST & 1 & 0,364 & 0,060 & 0,380 & $-0,365$ \\
TRIPLE HOP TEST & & 1 & 0,297 & $-0,353$ & $-0,076$ \\
CROSSOVER TEST & & 1 & 0,047 & 0,013 \\
COD & & & 1 & $0,498^{*}$ \\
SLJC & & & 1 \\
\hline COD_Cambio de dirección; SLCJ_Salto vertical unilateral con contra-movimiento & & \\
*_Correlación significativa entre test neuromusculares (p<0,05). & & & \\
\hline
\end{tabular}

La tabla 5 muestra el \% de coincidencia entre la PD (la de mayor rendimiento) de cada uno de los test.

Por último, resaltar que los valores de coincidencia entre la pierna descrita de forma subjetiva como dominante (la de chute) y la pierna descrita objetivamente como dominante (la de mayor rendimiento en cada prueba) fueron bajos. Los valores oscilaron entre el $41 \%$ (COD test y Triple Hop Test) y 59\% (Single hop test) (Tabla 6).

Tabla 5. Porcentaje de coincidencia entre pierna dominante (la de mayor rendimiento) según cada test.

\begin{tabular}{ccccc}
\hline & TRIPLE HOP & CROSSOVER & COD & SLCJ \\
& TEST & TEST & & \\
\hline SINGLE HOP TEST & $67 \%$ & $56 \%$ & $30 \%$ & $41 \%$ \\
TRIPLE HOP TEST & & $48 \%$ & $41 \%$ & $59 \%$ \\
CROSSOVER TEST & & & $44 \%$ & $59 \%$ \\
COD & & & $59 \%$ \\
\hline COD Cambio de dirección; SLCJ Salto vertical unilateral con contra-movimiento & &
\end{tabular}

Tabla 6. Porcentaje de coincidencia entre la pierna dominante establecida subjetivamente (la de chute) y la pierna dominante establecida objetivamente (la de mayor rendimiento).

\begin{tabular}{cccccc}
\hline & $\begin{array}{c}\text { SINGLE HOP } \\
\text { TEST }\end{array}$ & $\begin{array}{c}\text { TRIPLE HOP } \\
\text { TEST }\end{array}$ & CROSSOVER & COD & SLCJ \\
\hline $\begin{array}{c}\text { Test coinci- } \\
\text { dencia }\end{array}$ & $59 \%$ & $41 \%$ & $44 \%$ & $41 \%$ & $44 \%$ \\
\hline COD_Cambio de dirección; SLCJ_Salto vertical unilateral con contra-movimiento & & \\
\hline
\end{tabular}

\section{Discusión}

El objetivo del presente estudio fue comparar y cuantificar las asimetrías neuromusculares entre los miembros inferiores que presentan los jugadores de hockey sobre patines. Los principales hallazgos fueron que al comparar los resultados de la PD con los de la PND, existen diferencias estadísticamente significativas en todos los test neuromusculares registrados. En relación a los valores medios de ASI, éstos oscilaron entre el 4,93\% y el $9,60 \%$ en función del test. También destacar, que solo el test de SLCJ y el test de COD tuvieron una correlación significativa. Además, un 33,33\% de los jugadores obtuvieron un ASI superior al $10 \%$ en el test de SLCJ. 
Arboix-Alió, J.; Aguilera-Castells, J.; Rey-Abella, F.; Buscà, B., y Fort-Vanmeerhaeghe, A. (2018). Asimetrías neuromusculares entre miembros inferiores en jugadores de hockey sobre patines. RICYDE. Revista internacional de ciencias del deporte. 54(14), 358-373. https://doi.org/10.5232/ricyde2018.05406

En el presente estudio se observó que, comparando la PD con la PND, existen diferencias estadísticamente significativas en las cinco pruebas utilizadas. Parece ser que el hecho de obtener unos mejores registros con la PD (la de mayor rendimiento) podría estar determinado por la propia práctica deportiva (Maulder y Cronin, 2005), ya que las asimetrías entre extremidades reflejan las demandas del deporte practicado (Hewit y col., 2012). En el caso del hockey sobre patines, estas demandas específicas serían los constantes cambios de velocidad y de trayectoria, el desplazamiento asimétrico debido a la sujeción del stick o las acciones de pase y disparo. Estas demandas específicas, propias de dicho deporte y que vienen determinadas por la presencia del patinaje, la sujeción del stick y ejecutadas a gran velocidad, podrían conducir al desarrollo de adaptaciones asimétricas entre las extremidades. Actualmente no existen otros estudios que hayan estudiado las diferencias entre miembros inferiores en jugadores de hockey patines. Pese a ello si se han registrado diferencias significativas entre piernas en otros deportes de situación como fútbol (Ferrer-Roca, Balius, Domínguez-Castrillo, Linde, y Turmo-Garuz, 2014; Fousekis, Tsepis, y Vagenas, 2010), básquet (Sugiyama y col., 2014) o voleibol (Stephens y col., 2007).

Por lo que respecta al ASI difirió entre los test funcionales indicando que el porcentaje de asimetría puede variar dependiendo de la tarea utilizada para cuantificar el desequilibrio de las extremidades inferiores. En ninguno de los criterios ni en ningún test se encontró una media de ASI superior al $10 \%$, valor que si se obtiene en test de salto vertical indica riesgo incrementado de sufrir una lesión (Hewit y col., 2012; McElveen y col., 2010; Munro y Herrington, 2011). La media más alta se obtuvo en el test del SLCJ (9,60\%). Este valor sería muy similar a los reportados por Fort-Vanmeerhaeghe, Gual y col. (2016) (9,31\%), superior a los de Sugiyama y col., (2014) (6,3\%) e inferior a los de Thomas, Comfort, Dos'Santos, y Jones (2017) (12,26\%). En relación al "Hop Test", la media del valor ASI obtenida es de $6,35 \%$, valor superior a los reportados por Thomas y col., (2017) (4,97\%), Jones y Bampouras (2010) (4,47\%) y Meylan, Nosaka, Green, y Cronin (2010) (2,6\%). En el caso del COD test, Fort-Vanmeerhaeghe y col. (2015) indican un valor del 1,71\%, inferior al registro obtenido en este estudio (6,45\%). El hecho que el mayor ASI detectado se haya encontrado en el test de SLCJ coincide con Fort-Vanmeerhaeghe y col. (2015) los cuales indican que este test funcional probablemente reproduzca la tarea más sensible para detectar asimetrías entre las piernas.

Por otra parte, cuando se analiza el número de jugadores con un ASI $>10 \%$, valor que cuando es obtenido en pruebas de fuerza isocinética y de salto vertical puede incrementar el riesgo de lesión, se observa que también varía según el test. Concretamente, en el test de SLCJ nueve jugadores $(33,33 \%)$ se encontraban con unos valores de riesgo. Estos valores coincidirían con los reportados por Fort-Vanmeerhaeghe, Gual y col. (2016) con jugadoras de baloncesto $(34,21 \%)$ y con jugadores de voleibol $(31,7 \%)$. En el Single Hop Test y el Triple Hop Test, el porcentaje de jugadores de hockey sobre patines en riesgo sería más bajo; $18,51 \%$ y $11,11 \%$ respectivamente. Estos valores serían muy similares a los aportados por Troule y Casamichana (2016) en jugadores de fútbol con un 18,2\% y un 13,63\% en el Single HopTest y Triple Hop Test respectivamente. La literatura sugiere que un valor de ASI superior al $10 \%-15 \%$ en test de salto vertical podría correlacionarse con un mayor riesgo de sufrir una lesión. Pese a ello, aún faltan estudios longitudinales que demuestren la relación entre las asimetrías entre miembros inferiores y el riesgo de sufrir lesiones.

En relación a la correlación del ASI en los distintos test, existe una baja relación entre los valores de las diferentes capacidades neuromusculares. Únicamente se ha observado una correlación significativa entre el test de SLCJ y el COD test, que podría explicarse por la correlación que tienen las acciones de aceleración y de salto vertical (Balsalobre-Fernández, 
Arboix-Alió, J.; Aguilera-Castells, J.; Rey-Abella, F.; Buscà, B., y Fort-Vanmeerhaeghe, A. (2018). Asimetrías neuromusculares entre miembros inferiores en jugadores de hockey sobre patines. RICYDE. Revista internacional de ciencias del deporte. 54(14), 358-373. https://doi.org/10.5232/ricyde2018.05406

del Campo-Vecino, Tejero-González, y Alonso-Curiel, 2012; Shalfawi, Sabbah, Kailani, Tønnessen, y Enoksen, 2011). Referente a las otras pruebas funcionales, no se han obtenido correlaciones entre ellas, hecho que coincidiría con los resultados de Fort-Vanmeerhaeghe y col. (2015). Por lo tanto, con el objetivo de obtener un perfil neuromuscular completo de los atletas, es importante evaluarlos en diversas tareas (Fort-Vanmeerhaeghe y col., 2015). Del mismo modo, los programas de fortalecimiento, acondicionamiento y rehabilitación de los jugadores con el objetivo de controlar las asimetrías como factor de riesgo deberían centrarse en evaluar y entrenar las diferentes habilidades.

Otro hallazgo observado es que la coincidencia entre la clasificación subjetiva de la PD (pierna con la que se chuta un balón) y la clasificación objetiva de la PD (pierna con mayor rendimiento en cada prueba) es baja. Los resultados parecen indicar que no sería adecuado utilizar el criterio de identificación de la PD de forma subjetiva ya que la pierna con un mayor rendimiento dependerá de la especificidad de cada tarea/prueba realizada. Este hallazgo coincidiría y estaría en relación con el de otros autores (Ceroni y col., 2012; FortVanmeerhaeghe, Gual y col., 2016; Fort-Vanmeerhaeghe y col., 2015; Petisco, Carretero, y Sanchez-Sanchez, 2016). Aunque no hay investigaciones donde se examine la coincidencia entre la percepción de la PD de forma subjetiva y la PD de forma objetiva en hockey patines, estudios como los de Fort-Vanmeerhaeghe y col. (2015), exponen que, en 29 jugadoras de baloncesto, únicamente el $48 \%$ mostraron coincidencia entre ambos criterios en el test de SLCJ y en el test COD 52\%, valores muy similares también a los Ceroni y col. (2012) con una muestra de deportistas amateurs (51\%). En otros estudios realizados con jugadores de voleibol y de baloncesto, Fort-Vanmeerhaeghe, Gual y col. (2016) exponen que el porcentaje fue del 40\% en el test de SLCJ y Stephens, Lawson, DeVoe, y Reiser (2007) que fue del 50\%.

En cuanto a las limitaciones del presente estudio cabe señalar el reducido número de participantes estudiados. En futuros estudios, sería interesante ampliar la muestra y clasificarla según la posición de juego del deportista sobre la pista (portero, defensa o delantero), dado que las características físicas y físiológicas de la competición podrían depender, en parte, según la demarcación en la pista. También sería interesante de cara a futuros trabajos estudiar la influencia de la experiencia competitiva del deportista $o$ compararlo según las diferentes categorías y niveles deportivos presentes en el hockey patines. Finalmente, y con el objetivo de evidenciar la relación entre las asimetrías neuromusculares entre piernas y una mayor incidencia lesiva, se deberían llevar a cabo estudios longitudinales que puedan relacionar estas dos variables a lo largo de la temporada competitiva.

\section{Conclusiones}

Los registros obtenidos en el presente estudio muestran que el valor fisiológico de asimetría de los jugadores de hockey sobre patines oscila entre 4,93\% y $9,60 \%$ en función del test. Un total del 33,3\% de los jugadores presentaron un ASI > al 10\% en el test de salto vertical con contra-movimiento, situación que puede comportar un mayor riesgo de lesión.

Las asimetrías entre piernas no correlacionan en los diferentes test. Por ello el perfil neuromuscular completo entre piernas debería ser evaluado con las diferentes pruebas.

Por otra parte, la identificación subjetiva de la PD no es un criterio fiable para la selección de la pierna de mayor rendimiento, puesto que se ha observado una coincidencia baja. Por lo tanto, con el objetivo de calcular el \% de ASI, relacionado como posible factor de riesgo de lesión, se recomienda utilizar la pierna con mayor rendimiento de cada prueba como la dominante. 
Arboix-Alió, J.; Aguilera-Castells, J.; Rey-Abella, F.; Buscà, B., y Fort-Vanmeerhaeghe, A. (2018). Asimetrías neuromusculares entre miembros inferiores en jugadores de hockey sobre patines. RICYDE. Revista internacional de ciencias del deporte. 54(14), 358-373. https://doi.org/10.5232/ricyde2018.05406

\section{Referencias}

Arboix-Alió, J.; Aguilera-Castells, J., \& Ferrándiz, C. (2017). Roller hockey: correlative study about the speed capacity with and without skates. Revista Internacional de Deportes Colectivos, (31), 18-31.

Balsalobre-Fernández, C.; del Campo-Vecino, J.; Tejero-González, C. M., y Alonso-Curiel, D. (2012). Relación entre potencia máxima, fuerza máxima, salto vertical y sprint de 30 metros en atletas cuatrocentistas de alto rendimiento. Apunts Educación Física y Deportes, (108), 63-69.

https://doi.org/10.5672/apunts.2014-0983.es

Batista, A.; Bobo, M.; Lebre, E., y Ávila-Carvalho, L. (2015). Flexibilidad en gimnasia rítmica: asimetría funcional en gimnastas júnior portuguesas. Apunts Educación Física y Deportes, (120), 19-26.

https://doi.org/10.5672/apunts.2014-0983.es.(2015/2).120.03

Brumitt, J.; Heiderscheit, B. C.; Manske, R. C., \& Niemuth, P. E. (2013). Lower Extremity Functional Tests and Risk. The International Journal of Sports Physical Therapy, 8(3), 216-227.

Calleja-González, J.; Los Arcos, A.; Mejuto, G.; Casamichana, D.; San Román-Quintana, J., y Yanci, J. (2015). Reproducibilidad de test de aceleración y cambio de dirección en fútbol. RICYDE. Revista Internacional de Ciencias Del Deporte, 11(40), 104-115. http://dx.doi.org/10.5232/ricyde2015.04001

Carpes, F. P.; Mota, C. B., \& Faria, I. E. (2010). On the bilateral asymmetry during running and cycling - A review considering leg preference. Physical Therapy in Sport, 11(4), 136-142.

https://doi.org/10.1016/j.ptsp.2010.06.005

Castanharo, R.; Veras, M.; Alcantara, C.; Miana, A.; de Jesus, E.; Elias, J., \& Duarte, M. (2011). Asymmetries between lower limbs during jumping in female elite athletes from the brazilian national volleyball team. Portuguese Journal of Sport Sciences, $11(2), 53-56$.

Castillo-Rodríguez, A.; Fernández-García, J. C.; Chinchilla-Minguet, J. L., \& Carnero, E. Á. (2012). Relationship Between Muscular Strength and Sprints with Changes of Direction. Journal of Strength and Conditioning Research, 26(3), 725-732. https://doi.org/10.1519/JSC.0b013e31822602db

Ceroni, D.; Martin, X. E.; Delhumeau, C., \& Farpour-Lambert, N. J. (2012). Bilateral and Gender Differences During Single-Legged Vertical Jump Performance in Healthy Teenagers. Journal of Strength and Conditioning Research, 26(2), 452-457. https://doi.org/10.1519/JSC.0b013e31822600c9

Cohen, J. (1988). Statistical power analysis for behavioural science. NJ: Lawrence Erlbaum.

Daneshjoo, A.; Rahnama, N.; Mokhtar, A. H., \& Yusof, A. (2013). Bilateral and unilateral asymmetries of isokinetic strength and flexibility in male young professional soccer players. Journal of Human Kinetics, 36, 45-53.

https://doi.org/10.2478/hukin-2013-0005

Dauty, M.; Menu, P.; Fouasson-chailloux, A.; Ferréol, S., \& Dubois, C. (2016). Prediction of hamstring injury in professional soccer players by isokinetic measurements. Muscles, Ligaments and Tendons, 6(1), 116-123.

https://doi.org/10.11138/mltj/2016.6.1.116 
Arboix-Alió, J.; Aguilera-Castells, J.; Rey-Abella, F.; Buscà, B., y Fort-Vanmeerhaeghe, A. (2018). Asimetrías neuromusculares entre miembros inferiores en jugadores de hockey sobre patines. RICYDE. Revista internacional de ciencias del deporte. 54(14), 358-373. https://doi.org/10.5232/ricyde2018.05406

de Blas, X.; Padullés, J. M.; López del Amo, J. L., \& Guerra-Balic, M. (2012). Creation and Validation of Chronojump-Boscosystem: A Free Tool to Measure Vertical Jumps. RICYDE. Revista Internacional de Ciencias Del Deporte, 8(30), 334-356. https://doi.org/10.5232/ricyde2012.03004

Dellagrana, R. A.; Diefenthaeler, F.; Carpes, F. P.; Hernandez, S. G., \& de Campos, W. (2015). Evidence for isokinetic knee torque asymmetries in male long distance-trained runners. International Journal of Sports Physical Therapy, 10(4), 514-9.

Ferrer-Roca, V.; Balius, X.; Domínguez-Castrillo, O.; Linde, F. J., y Turmo-Garuz, A. (2014). Evaluación de factores de riesgo de lesión del ligamento cruzado anterior en jugadores de fútbol de alto nivel. Apunts Medicina de l'Esport, 48(181), 5-10. https://doi.org/10.1016/j.apunts.2013.06.003

Fort-Vanmeerhaeghe, A.; Gual, G.; Romero-Rodriguez, D., \& Unnitha, V. (2016). Lower limb neuromuscular asymmetry in volleyball and basketball players. Journal of Human Kinetics, 50(1), 135-143.

https://doi.org/10.1515/hukin-2015-0150

Fort-Vanmeerhaeghe, A.; Montalvo, A. M.; Sitjà-Rabert, M.; Kiefer, A. W., \& Myer, G. D. (2015). Neuromuscular asymmetries in the lower limbs of elite female youth basketball players and the application of the skillful limb model of comparison. Physical Therapy in Sport, 16(4), 317-323.

https://doi.org/10.1016/j.ptsp.2015.01.003

Fort-Vanmeerhaeghe, A.; Rodriguez, D. R.; Tutusaus, L. C.; Calafat, C. B.; Riera, M. L., y Vidal, A. M. (2009). Diferencias en la estabilidad postural estática y dinámica según sexo y pierna dominante. Apunts Medicina de l'Esport, 44(162), 74-81. https://doi.org/10.1016/S1886-6581(09)70112-4

Fort-Vanmeerhaeghe, A.; Romero-Rodriguez, D.; Montalvo, A. M.; Kiefer, A. W.; Lloyd, R. S., \& Myer, G. D. (2016). Integrative Neuromuscular Training and Injury Prevention in Youth Athletes. Part I. Strength and Conditioning Journal, 38(3), 36-48. https://doi.org/10.1519/SSC.0000000000000229

Fort-Vanmeerhaeghe, A., y Romero, D. (2013). Análisis de los factores de riesgo neuromusculares de las lesiones deportivas. Apunts Medicina de l'Esport, 48(179).

Fousekis, K.; Tsepis, E., \& Vagenas, G. (2010). Lower limb strength in professional soccer players: profile, asymmetry, and training age. Journal of Sports Science \& Medicine, 9(3), 364-73.

García, C.; Albaladejo, R.; Villanueva, R., y Navarro, E. (2015). Deporte de ocio en España. epidemiología de las lesiones y sus consecuencias. Apunts. Educación Física Y Deportes, 1(119), 62-70. https://doi.org/http://dx.doi.org/10.5672/apunts.2014-0983.es.(2015/1).119.03

Griffin, L. Y.; Albohm, M. J.; Arendt, E. A.; Bahr, R.; Beynnon, B. D.; DeMaio, M.; ... Yu, B. (2006). Understanding and preventing noncontact anterior cruciate ligament injuries. The American Journal of Sports Medicine, 34(9), 1512-1532. https://doi.org/10.1177/0363546506286866

Haahr, M. (2017). True random number service. Random.org. Recuperado 10 de febrero del 2017, a partir de: http://www.random.org/nform.htm

Hart, N. H.; Nimphius, S.; Weber, J.; Spiteri, T.; Rantalainen, T.; Dobbin, M., \& Newton, R. U. (2016). Musculoskeletal Asymmetry in Football Athletes: A Product of Limb Function over Time. Medicine and Science in Sports and Exercise, 48(7), 1379-1387. https://doi.org/10.1249/MSS.0000000000000897 
Arboix-Alió, J.; Aguilera-Castells, J.; Rey-Abella, F.; Buscà, B., y Fort-Vanmeerhaeghe, A. (2018). Asimetrías neuromusculares entre miembros inferiores en jugadores de hockey sobre patines. RICYDE. Revista internacional de ciencias del deporte. 54(14), 358-373. https://doi.org/10.5232/ricyde2018.05406

Hewit, J.; Cronin, J., \& Hume, P. (2012). Multidirectional Leg Asymmetry Assessment in Sport. Strength and Conditioning Journal, 34(1), 82-86. https://doi.org/10.1519/SSC.0b013e31823e83db

Impellizzeri, F. M.; Rampinini, E.; Maffiuletti, N., \& Marcora, S. M. (2007). A vertical jump force test for assessing bilateral strength asymmetry in athletes. Medicine and Science in Sports and Exercise, 39(11), 2044-2050. https://doi.org/10.1249/mss.0b013e31814fb55c

Jones, P. A., \& Bampouras, T. M. (2010). A Comparison of Isokinetic and Functional Methods of Assessing Bilateral Strength Imbalance. Journal of Strength and Conditioning Research, 24(6), 1553-1558. https://doi.org/10.1519/JSC.0b013e3181dc4392

Krzykała, M., \& Leszczyński, P. (2015). Asymmetry in body composition in female hockey players. Homo: Journal of Comparative Human Biology, 66(4), 379-86. https://doi.org/10.1016/j.jchb.2015.02.008

Logerstedt, D. S.; Snyder-Mackler, L.; Ritter, R. C.; Axe, M. J., \& Godges, J. J. (2010). Knee Stability and Movement Coordination Impairments: Knee Ligament Sprain. Journal of Orthopaedic \& Sports Physical Therapy, 40(4), A1-A37. https://doi.org/10.2519/jospt.2010.0303

Markou, S., \& Vagenas, G. (2006). Multivariate isokinetic asymmetry of the knee and shoulder in elite volleyball players. European Journal of Sport Science, 6(March), 7180. https://doi.org/10.1080/17461390500533147

Marshall, B.; Franklyn-Miller, A.; Moran, K.; King, E.; Richter, C.; Gore, S.; ... Falvey, É. (2015). Biomechanical symmetry in elite rugby union players during dynamic tasks: an investigation using discrete and continuous data analysis techniques. BMC Sports Science, Medicine and Rehabilitation, 7(1), 13. https://doi.org/10.1186/s13102-015-0006-9

Maulder, P., \& Cronin, J. (2005). Horizontal and vertical jump assessment: reliability, symmetry, discriminative and predictive ability. Physical Therapy in Sport, 6(2), 7482. https://doi.org/10.1016/j.ptsp.2005.01.001

McElveen, M. T.; Riemann, B. L., \& Davies, G. J. (2010). Bilateral Comparison of Propulsion Mechanics During Single-Leg Vertical Jumping. Journal of Strength and Conditioning Research, 24(2), 375-381. https://doi.org/10.1519/JSC.0b013e3181c06e0b

Menzel, H.-J.; Chagas, M. H.; Szmuchrowski, L. A.; Araujo, S. R. S.; de Andrade, A. G. P., \& de Jesus-Moraleida, F. R. (2013). Analysis of Lower Limb Asymmetries by Isokinetic and Vertical Jump Tests in Soccer Players. Journal of Strength and Conditioning Research, 27(5), 1370-1377. https://doi.org/10.1519/JSC.0b013e318265a3c8

Meylan, C.; McMaster, T.; Cronin, J.; Mohammad, N. I.; Rogers, C., \& DeKlerk, M. (2009). Single-Leg Lateral, Horizontal, and Vertical Jump Assessment: Reliability, Interrelationships, and Ability to Predict Sprint and Change-of-Direction Performance. Journal of Strength and Conditioning Research, 23(4), 1140-1147. https://doi.org/10.1519/JSC.0b013e318190f9c2

Meylan, C.; Nosaka, K.; Green, J., \& Cronin, J. B. (2010). Temporal and kinetic analysis of unilateral jumping in the vertical, horizontal, and lateral directions. Journal of Sports Sciences, 28(5), 545-554.

https://doi.org/10.1080/02640411003628048 
Arboix-Alió, J.; Aguilera-Castells, J.; Rey-Abella, F.; Buscà, B., y Fort-Vanmeerhaeghe, A. (2018). Asimetrías neuromusculares entre miembros inferiores en jugadores de hockey sobre patines. RICYDE. Revista internacional de ciencias del deporte. 54(14), 358-373. https://doi.org/10.5232/ricyde2018.05406

Miyaguchi, K., \& Demura, S. (2010). Specific Factors That Influence Deciding the Takeoff Leg during Jumping Movements. Journal of Strength and Conditioning Research, 24(9), 2516-2522.

https://doi.org/10.1519/JSC.0b013e3181e380b5

Munro, A. G., \& Herrington, L. C. (2011). Between-Session Reliability of Four Hop Tests and the Agility T-Test. Journal of Strength and Conditioning Research, 25(5), 14701477. https://doi.org/10.1519/JSC.0b013e3181d83335

Murphy, D. F.; Connolly, D. a J., \& Beynnon, B. D. (2003). Risk factors for lower extremity injury: a review of the literature. British Journal of Sports Medicine, 37(1), 13-29.

https://doi.org/10.1136/bjsm.37.1.13

Noyes, F. R.; Barber, S. D., \& Mangine, R. E. (1991). Abnormal lower limb symmetry determined by function hop tests after anterior cruciate ligament rupture. The American Journal of Sports Medicine, 19(5), 513-8.

Overmoyer, G. V., \& Reiser, R. F. (2013). Relationships Between Asymmetries in Functional Movements and the Star Excursion Balance Test. Journal of Strength and Conditioning Research, 27(7), 2013-2024.

https://doi.org/10.1519/JSC.0b013e3182779962

Paterno, M. V.; Schmitt, L. C.; Ford, K. R.; Rauh, M. J.; Myer, G. D.; Huang, B., \& Hewett, T. E. (2010). Biomechanical measures during landing and postural stability predict second anterior cruciate ligament injury after anterior cruciate ligament reconstruction and return to sport. The American Journal of Sports Medicine, 38(10), 1968-1978.

https://doi.org/10.1177/0363546510376053

Petisco, C.; Carretero, M., y Sanchez-Sanchez, J. (2016). ¿Es el ejercicio físico un factor determinante de las asimetrías funcionales en la extremidad inferior? Apunts. Educación Fisica y Deportes, 125, 7-20. https://doi.org/10.5672/apunts.2014-0983.es.(2016/3).125.01

Pons, M., y Ferrer, H. (1991). Lesiones, traumatologicas en el hockey sobre patines. Apunts: Educació Física i Esports, (23), 35-44.

Pontaga, I., \& Zidens, J. (2014). Shoulder rotator muscle dynamometry characteristics: side asymmetry and correlations with ball-throwing speed in adolescent handball players. Journal of Human Kinetics, 42, 41-50.

https://doi.org/10.2478/hukin-2014-0059

Porta, J.; Bescós, R., \& Vallejo, L. (2009). The Anthropometric Method Versus Different Bia Systems to Estimate Fat in Athletes. Archivos de Medicina Del Deporte, XXVI(131), 187-193.

Rahnama, N.; Lees, A., \& Bambaecichi, E. (2005). Comparison of muscle strength and flexibility between the preferred and non-preferred leg in English soccer players. Ergonomics, 48(11-14), 1568-1575. https://doi.org/10.1080/00140130500101585

Reid, A.; Birmingham, T. B.; Stratford, P. W.; Alcock, G. K., \& Giffin, J. R. (2007). Hop testing provides a reliable and valid outcome measure during rehabilitation after anterior cruciate ligament reconstruction. Physical Therapy, 87(3), 337-349. https://doi.org/10.2522/ptj.20060143

Ross, S. E., \& Guskiewicz, K. M. (2004). Examination of static and dynamic postural stability in individuals with functionally stable and unstable ankles. Clinical Journal of Sport Medicine, 14(6), 332-338.

https://doi.org/10.1097/00042752-200411000-00002 
Arboix-Alió, J.; Aguilera-Castells, J.; Rey-Abella, F.; Buscà, B., y Fort-Vanmeerhaeghe, A. (2018). Asimetrías neuromusculares entre miembros inferiores en jugadores de hockey sobre patines. RICYDE. Revista internacional de ciencias del deporte. 54(14), 358-373. https://doi.org/10.5232/ricyde2018.05406

Ross, S.; Guskiewicz, K.; Prentice, W.; Schneider, R., \& Yu, B. (2004). Comparison of Biomechanical Factors between the Kicking and Stance Limbs. Journal of Sport Rehabilitation, 13(2), 135-150.

https://doi.org/10.1123/jsr.13.2.135

Sannicandro, I.; Cofano, G.; Rosa, R. A., \& Piccinno, A. (2014). Balance training exercises decrease lower-limb strength asymmetry in young tennis players. Journal of Sports Science \& Medicine, 13(2), 397-402.

Schiltz, M.; Lehance, C.; Maquet, D.; Bury, T.; Crielaard, J.-M., \& Croisier, J.-L. (2009). Explosive Strength Imbalances in Professional Basketball Players. Journal of Athletic Training, 44(1), 39-47.

https://doi.org/10.4085/1062-6050-44.1.39

Shalfawi, S. A.; Sabbah, A.; Kailani, G.; Tønnessen, E., \& Enoksen, E. (2011). The Relationship Between Running Speed and Measures of Vertical Jump in Professional Basketball Players: A Field-Test Approach. Journal of Strength and Conditioning Research, 25(11), 3088-3092. https://doi.org/10.1519/JSC.0b013e318212db0e

Simas Frutuoso, A.; Diefenthaeler, F.; Vaz, M. A., \& de la Rocha Freitas, C. (2016). Lower limb asymmetries in rhythmic gymnastics athletes. International Journal of Sports Physical Therapy, 11(1), 34-43.

Stephens, T. M.; Lawson, B. R.; DeVoe, D. E., \& Reiser, R. F. (2007). Gender and bilateral differences in single-leg countermovement jump performance with comparison to a double-leg jump. Journal of Applied Biomechanics, 23(3), 190-202.

Sugiyama, T.; Kameda, M.; Kageyama, M.; Kiba, K.; Kanehisa, H., \& Maeda, A. (2014). Asymmetry between the Dominant and Non-Dominant Legs in the Kinematics of the Lower Extremities during a Running Single Leg Jump in Collegiate Basketball Players. Journal of Sports Science \& Medicine, 13(4), 951-7.

Theoharopoulos, A.; Tsitskaris, G.; Nikopoulou, M., \& Tsaklis, P. (2000). Knee Strength of Professional Basketball Players. The Journal of Strength and Conditioning Research, 14(4), 457.

https://doi.org/10.1519/1533-4287(2000)014<0457:KSOPBP>2.0.CO;2

Thomas, C.; Comfort, P.; Dos'Santos, T., \& Jones, P. (2017). Determining Bilateral Strength Imbalances in Youth Basketball Athletes. International Journal of Sports Medicine, 38(9), 683-690.

https://doi.org/10.1055/s-0043-112340

Troule, S., y Casamichana, D. (2016). Aplicación de pruebas funcionales para la detección de asimetrías en jugadores de fútbol. Journal of Sport and Health Research, 8(1), 53-64.

Varlotta, G. P.; Lager, S. L.; Nicholas, S.; Browne, M., \& Schlifstein, T. (2000). Professional roller hockey injuries. Clinical Journal of Sport Medicine: Official Journal of the Canadian Academy of Sport Medicine, 10(1), 29-33.

Yanci, J., \& Camara, J. (2016). Bilateral and unilateral vertical ground reaction forces and leg asymmetries in soccer players. Biology of Sport, 33(2), 179-183. https://doi.org/10.5604/20831862.1198638 